\title{
Ki-67: a useful marker for the evaluation of dysplasia in ulcerative colitis
}

\author{
S N Andersen, T O Rognum, A Bakka, O P F Clausen
}

\begin{abstract}
Aims-Evaluation of dysplasia in long standing ulcerative colitis is a difficult and often subjective task. Therefore, the aim of this study was to search for a more objective parameter to help distinguish regenerative changes from epithelial dysplasia.

Methods-A total of 97 sections from colectomy specimens from 12 patients with ulcerative colitis of more than 10 years duration were stained immunohistochemically with MIB 1 to detect differences in the frequency and pattern of nuclei positive for the proliferation marker Ki-67. All patients had epithelial dysplasia in one or more areas (high grade dysplasia, $n=16$; low grade dysplasia, $\mathrm{n}=15$; indefinite for dysplasia, $\mathbf{n}=16$ ), and three patients had additional adenocarcinoma (one Dukes's C multifocal, mucinous carcinoma; one Dukes's $C$ adenocarcinoma in the sigmoid; and one Dukes's A adenocarcinoma in the caecum). Two patients had adenomas-one had an $8 \mathrm{~cm}$ villous adenoma with intramucosal carcinoma, and the other had a $4 \mathrm{~cm}$ tubulovillous adenoma with high grade dysplasia.
\end{abstract}

Results-There were highly significant differences between the percentages of Ki-67 immunopositive cells in low grade and high grade dysplasia and carcinoma compared with regenerative epithelium. In high grade dysplasia and carcinoma, the distribution of $\mathrm{Ki}-67$ positive cells was diffuse throughout the full length of the crypt, whereas low grade dysplasia and epithelium indefinite for dysplasia, as well as regenerative epithelium, showed an expanded basal zone.

Conclusions-Assessment of the number of Ki-67 immunostained cells is of additional value in deciding whether the mucosa is regenerative or dysplastic, and the MIB 1 staining pattern is characteristic for most lesions with high grade dysplasia and carcinoma. Therefore, this technique could be combined with routine histological evaluation of colorectal epithelium being examined for dysplasia. (F Clin Pathol: Mol Pathol 1998;51:327-332)

Keywords: Ki-67 immunohistochemistry; ulcerative colitis; dysplasia

Extensive and long standing ulcerative colitis of more than eight years duration is well known to predispose patients to the development of colorectal carcinoma. ${ }^{1-4}$ This increase in the incidence of cancer might be related to increased cellular proliferation within the mucosal crypts as a result of recurrent or persistent inflammation, and the risk of cancer increases with the duration of the disease. ${ }^{5}$ In time, inflammation may give rise to epithelial changes known as dysplasia. Despite attempts to standardise the criteria for the classification of dysplasia, ${ }^{6}$ evaluation and grading of dysplasia in the colorectum is a difficult and partly subjective task. Epithelial regeneration in inflammation can be irregular or bizarre and imitate dysplasia; or even worse, disguise the neoplastic or preneoplastic changes.

In previous studies, the use of tritiated thymidine and the non-radioactive thymidine analogue, bromodeoxyuridine, has demonstrated increased labelling indices in apparently normal rectal mucosa in patients at high risk of developing colorectal carcinoma. ${ }^{78}$ The monoclonal antibody $\mathrm{Ki}-67$ detects a nuclear antigen that reflects cell proliferation, thus identifying the growth fraction of tissues and tumours. ${ }^{9}$ Recently, Ki-67 like antibodies (MIB 1-3) were developed that are reactive in sections of formalin fixed, paraffin wax embedded tissue after antigen retrieval. ${ }^{10-12}$

Shepherd and colleagues ${ }^{13}$ evaluated the growth fraction in colorectal carcinomas using frozen sections and this was followed by several studies of carcinomas and adenomas. ${ }^{14-17}$ Until recently, there have been few studies of $\mathrm{Ki}-67$ antigen in relation to regeneration and dysplasia in ulcerative colitis. ${ }^{11}{ }^{19}$ The aim of our study was to try to validate these results.

Our material comprised 30 colectomy specimens collected from patients with ulcerative colitis of long duration-most of them having epithelial dysplasia, and some with carcinoma. This gave us an opportunity to study $\mathrm{Ki}-67$ reactivity in epithelial changes from regeneration through low and high grade dysplasia to carcinoma, and offered important information about proliferation related changes that might be of prognostic importance during successive stages of epithelial transformation.

\section{Methods}

PATIENTS AND COLECTOMY SPECIMENS

From our total of 30 colectomy specimens, 12 patients who had undergone coloproctectomy between June 1992 and September 1994 were chosen. These patients suffered from long standing (10-30 years) ulcerative colitis with different types of epithelial changes, including dysplasia and cancer. All specimens showed more or less inactive colitis with little active inflammation. Of these 12 patients, 10 were men and two were women, (median age, 41 years; range, 29-75). Three had 
Table 1 Clinical and pathological data of patients with ulcerative colitis

\begin{tabular}{|c|c|c|c|c|c|c|}
\hline Patient & Age & $\operatorname{Sex}$ & $\begin{array}{l}\text { Duration } \\
\text { (years) }\end{array}$ & $\begin{array}{l}\text { Carcinoma Dukes's } \\
\text { stage/other tumour }\end{array}$ & $\begin{array}{l}\text { Localisation } \\
\text { of tumour }\end{array}$ & $\begin{array}{l}\text { Max. grade of } \\
\text { dysplasia }\end{array}$ \\
\hline 1 & 75 & M1 & 16 & C multifocal & $\mathrm{T} / \mathrm{L}$ colon & High grade \\
\hline 2 & 41 & $M$ & 25 & & Sigmoid & Low grade \\
\hline 3 & 31 & M & 16 & A & Caecum & High grade \\
\hline 4 & 40 & $M$ & 26 & V tumour intramuc. carc. & $\mathrm{T}$ colon & Low grade \\
\hline 5 & 64 & $\mathrm{~F}$ & 13 & Tub. high grade & Rectum & High grade \\
\hline 6 & 41 & $M$ & 11 & & & High grade \\
\hline 7 & 39 & $M$ & 16 & & & High grade \\
\hline 8 & 41 & M & 24 & & & High grade \\
\hline 9 & 46 & M & 30 & & & High grade \\
\hline 10 & 38 & $\mathrm{M}$ & 20 & & & Low grade \\
\hline 11 & 45 & $M$ & 10 & & & Low grade \\
\hline 12 & 29 & $\mathrm{~F}$ & 15 & & & Low grade \\
\hline
\end{tabular}

A, Dukes's stage A adenocarcinoma; C, Dukes's stage C adenocarcinoma; T/L colon, transverse and descendant colon; $\mathrm{T}$ colon, transverse colon; Tub, tubulovillous adenoma; $\mathrm{V}$ tumour intramuc. carc., villous adenoma with intramucosal carcinoma.

adenocarcinomas-one had a Dukes's A carcinoma in the caecum, another had Dukes's C carcinoma in the sigmoid, and the third patient presented with a multifocal and mucinous Dukes's C carcinoma with three tumours in the transverse and descendant colon. One patient had a large, villous adenoma (diameter, $8 \mathrm{~cm}$ ), with intramucosal carcinoma in the transverse colon, and one patient had a tubulovillous adenoma $(4 \mathrm{~cm})$ with high grade dysplasia in the rectum (table 1 ).

\section{IMMUNOHISTOCHEMICAL ANALYSIS}

On average, 25 sections were taken throughout the large bowel wall of each colectomy specimen. These were fixed in buffered formalin and stained with a trichrome routine method containing haematoxylin, azofloxine, and saffron. ${ }^{20}$ Based on light microscopy, seven to 10 sections were chosen to cover the spectrum of epithelial alterations in each patient, a total of 97 sections altogether. No area was found to be completely normal in any of the colectomy specimens. Therefore, normal control mucosa was scored in eight biopsies from patients who had undergone colonoscopy because of uncharacteristic bowel problems, but who did not have mucosal changes. There were 43 sections with regenerative epithelium. The other groups were: "epithelium indefinite for dysplasia, probably positive" $(n=16)$; low grade dysplasia $(n=15)$; and high grade dysplasia $(n=16)$. From five carcinomas in three patients, seven sections were taken (table 2 ). There was little active inflammation, and all sections, except those from carcinomas and adenomas, showed atrophy of the mucosa, with branched crypts and some chronic inflammation.

Serial sections cut at $5 \mu \mathrm{m}$ thickness were obtained for immunohistochemical evaluation and placed on pretreated, gelatine coated slides. After deparaffinising with xylene, the sections were rehydrated through graded alco-

Table 2 Epithelial changes and Ki-67 immunoreactivity in patients with ulcerative colitis

\begin{tabular}{lllllc}
\hline Histological classification & $\begin{array}{l}\text { No. of } \\
\text { sections }\end{array}$ & Basal & $\begin{array}{l}\text { Expanded } \\
\text { basal zone }\end{array}$ & $\begin{array}{l}\text { Extension to } \\
\text { surface }\end{array}$ & Diffuse \\
\hline Regenerative & 43 & 0 & 43 & 0 & 0 \\
Indefinite for dysplasia & 16 & 0 & 14 & 2 & 0 \\
Low grade dysplasia & 15 & 0 & 11 & 3 & 1 \\
High grade dysplasia & 16 & 0 & 3 & 2 & 11 \\
Carcinoma & 7 & 0 & 0 & 0 & 7 \\
Total & 97 & & & & \\
\hline
\end{tabular}

hols to water. The slides were then incubated in $10 \mathrm{mM}$ citrate buffer ( $\mathrm{pH}$ 6.3) in a microwave oven $(750 \mathrm{~W})$ for three periods of five minutes. After incubation, the sections were cooled for 30 minutes at room temperature, and then washed in Tris buffer for five minutes. The sections were then incubated overnight (18 hours) at room temperature with the mouse monoclonal antibody MIB 1 (a gift from Dr J Gerdes, Germany). This antibody recognises Ki-67 epitopes in formalin fixed material. Both positive and negative (omitting primary antibody) control reactions for each series of immunostained sections were carried out. Staining was performed with the conventional alkaline phosphatase anti-alkaline phosphatase method, using fast red (Sigma Chemical Co, St Louis, Missouri, USA) as chromogen. The slides were lightly counterstained with haematoxylin.

Cells were scored as positive when nuclear staining was evident. Slides were chosen where crypts were well orientated, and the total number of cells in whole crypts was always counted up to the mucosal surface. The number of crypts included depended on the grade of atrophy in that definite region. A pilot experiment showed that counting of about 500 cells gave a valid percentage of positive cells that did not change significantly from that of scoring up to 1000 cells. The percentage of positive cells was registered for each region and tabulated. In addition, we classified each lesion according to the pattern of $\mathrm{Ki}-67$ immunoreactivity. We distinguished between four patterns: basal, when the proliferating cells were confined to the basal one third of the crypt; expanded basal zone, when Ki-67 positive cells extended beyond the basal one third of the crypt; extension to surface, when positive cells presented on the mucosal surface; and diffuse, when proliferating cells were present at all levels within the crypt, as well as on the mucosal surface. These are similar to the categories proposed by Noffsinger et al. ${ }^{19}$

\section{STATISTICAL METHODS}

To account for possible correlations between observations from the same patient, we used a variance component model with "patient" as random component and "epithelial grading" as "fixed effect". We used the "proc mixed" procedure of the SAS statistical software package. ${ }^{21}$ The $p$ values were Bonferroni corrected. The Kappa test ${ }^{22}$ was used to test for intraobserver and interobserver reproducibility. A p value $<0.05$ was considered to be significant.

\section{Results}

EVALUATION OF EPITHELIAL CHANGES

Two independent and experienced histopathologists (SNA and OPFC) evaluated independently 59 of the 97 routinely stained slides for different grades of dysplasia according to standard criteria for comparison. ${ }^{6}$ The remainder of the slides were classified by SNA alone and afterwards agreed upon by OPFC. Interobserver agreement was substantial $($ Kappa $=0.64)$, and intraobserver agreement 

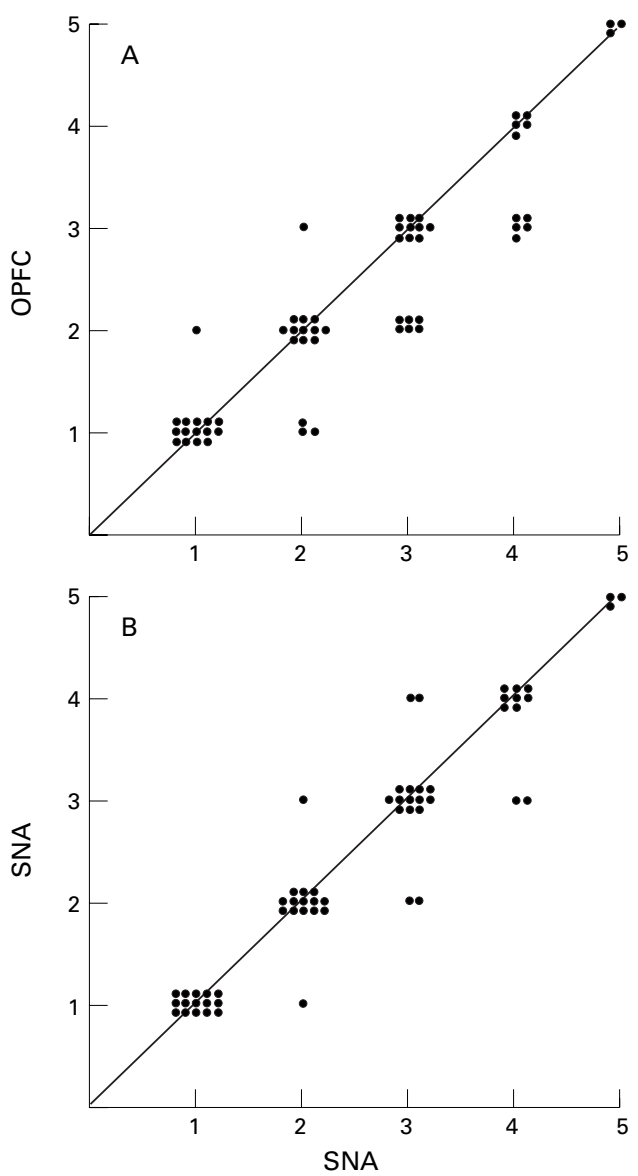

Figure 1 Scatter diagrams of interobserver and intraobserver reproducibility. The degree of accordance for grading of epithelial changes $(A)$ between the first observer $S N A$ and the second observer OPFC $\left(P_{o}=0.73\right.$;

Kappa $=0.64)$, and $(B)$ between two observations by the same histopathologist ( $S N A$ ), the last performed blindly after 12 weeks $\left(P_{o}=0.86\right.$; Kappa $\left.=0.82\right)$.

after three months was "almost perfect" $($ Kappa $=0.82)($ fig 1$)$.

COUNTING OF KI-67 POSITIVE CELLS

Of the 97 sections, 43 showed regenerative changes with little active inflammation, 16 were indefinite for dysplasia, 15 showed low grade

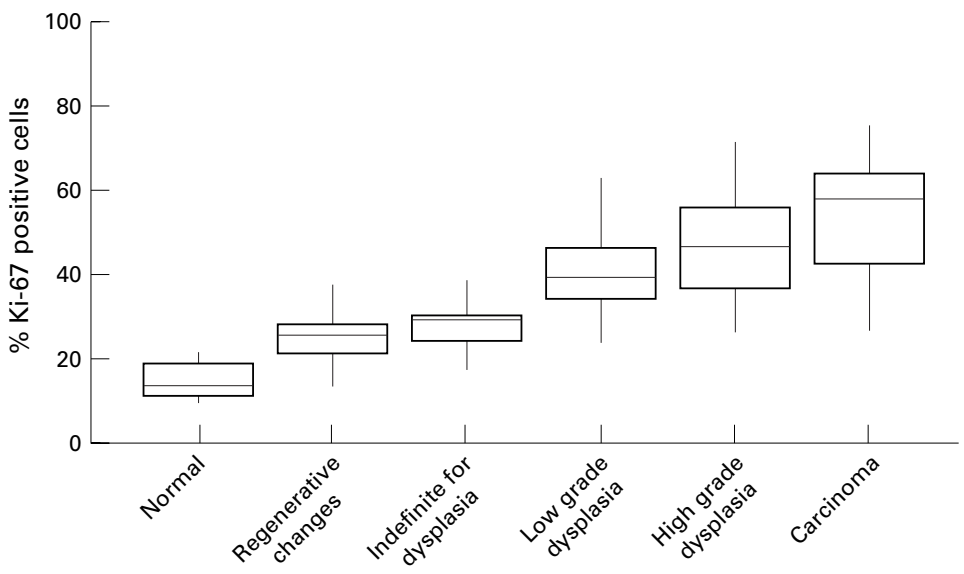

Figure 2 Box plot illustrating percentage of Ki-67 positive cells in different epithelial lesions in ulcerative colitis: normal epithelium, regenerative epithelium with little active inflammation, epithelium indefinite for dysplasia, low grade dysplasia, high grade dysplasia, and carcinoma. The horizontal lines in the boxes indicate the mean. dysplasia, 16 showed high grade dysplasia, and seven slides were from the five carcinomas examined.

The sections showed varying numbers of cells with positive nuclear staining (table 2) and negligible background staining. Most cells stained strongly positive, and less than $10 \%$ on average were stained less intensely. All cells with staining intensity above background were scored as positive for $\mathrm{Ki}-67$. The statistical analysis showed that the groups differed significantly ( $\mathrm{p}$ values $<0.001$ ). We calculated the Bonferroni corrected comparison between groups. The numbers of $\mathrm{Ki}-67$ positive cells were significantly higher in areas with low grade dysplasia (mean (SD), 41.6 (12)), high grade dysplasia (mean (SD), 47.4 (13)), and carcinoma (mean (SD) 53.9 (17)) compared with regenerative epithelium (mean (SD), 25.7 (6)), (all p values < 0.001; fig 2), as well as low grade dysplasia, high grade dysplasia, and carcinoma compared with normal epithelium (mean (SD) 14.8 (4.3); $\mathrm{p}<0.001$ ). Epithelium with low grade dysplasia had significantly higher numbers of immunopositive cells compared with both epithelium indefinite for dysplasia and regenerative epithelium $(\mathrm{p}<0.001)$, whereas the number of $\mathrm{Ki}-67$ positive cells in epithelium indefinite for dysplasia was not statistically different from that in regenerative epithelium $(p \approx 1)$. Epithelium indefinite for dysplasia had significantly more positively stained nuclei compared with normal epithelium $(\mathrm{p}=0.009)$. Comparing high grade dysplasia with low grade gave a $\mathrm{p}$ value of 0.19 , and positive staining in high grade dysplasia was almost equal to that in carcinoma. Carcinoma differed from low grade dysplasia ( $p=0.03)$, as did regenerative compared with normal epithelium $(p=0.03)$. Examples of positive staining with MIB 1 in regenerative epithelium, in low and high grade dysplasia, and in carcinoma are shown in fig 3.

\section{KI-67 STAINING PATTERNS}

Table 2 shows patterns of Ki-67 immunoreactivity in non-neoplastic and neoplastic lesions. All sections with regenerative changes had expansion of the basal zone (43 of 43) compared with that which is normally seen in colonic mucosa. Most high grade dysplasias (11 of 16) and all sections from carcinomas (7 of 7) showed diffuse and scattered staining along the full length of the crypts. The remainder of the high grade dysplasias showed either extension of staining to the surface ( 2 of 16$)$ or an expanded basal zone ( 3 of 16 ). In both low grade dysplasia and epithelium indefinite for dysplasia, most lesions had an expanded basal zone (11 of 15 and 14 of 16, respectively). Three lesions with low grade dysplasia had extension of $\mathrm{Ki}-67$ positive cells up to and in to the surface epithelium, and one single lesion showed diffuse staining along the crypts.

\section{Discussion}

The main finding of our study was the highly significant differences in percentages of cells with Ki-67 immunostaining in high grade and low grade dysplastic lesions and carcinoma, 

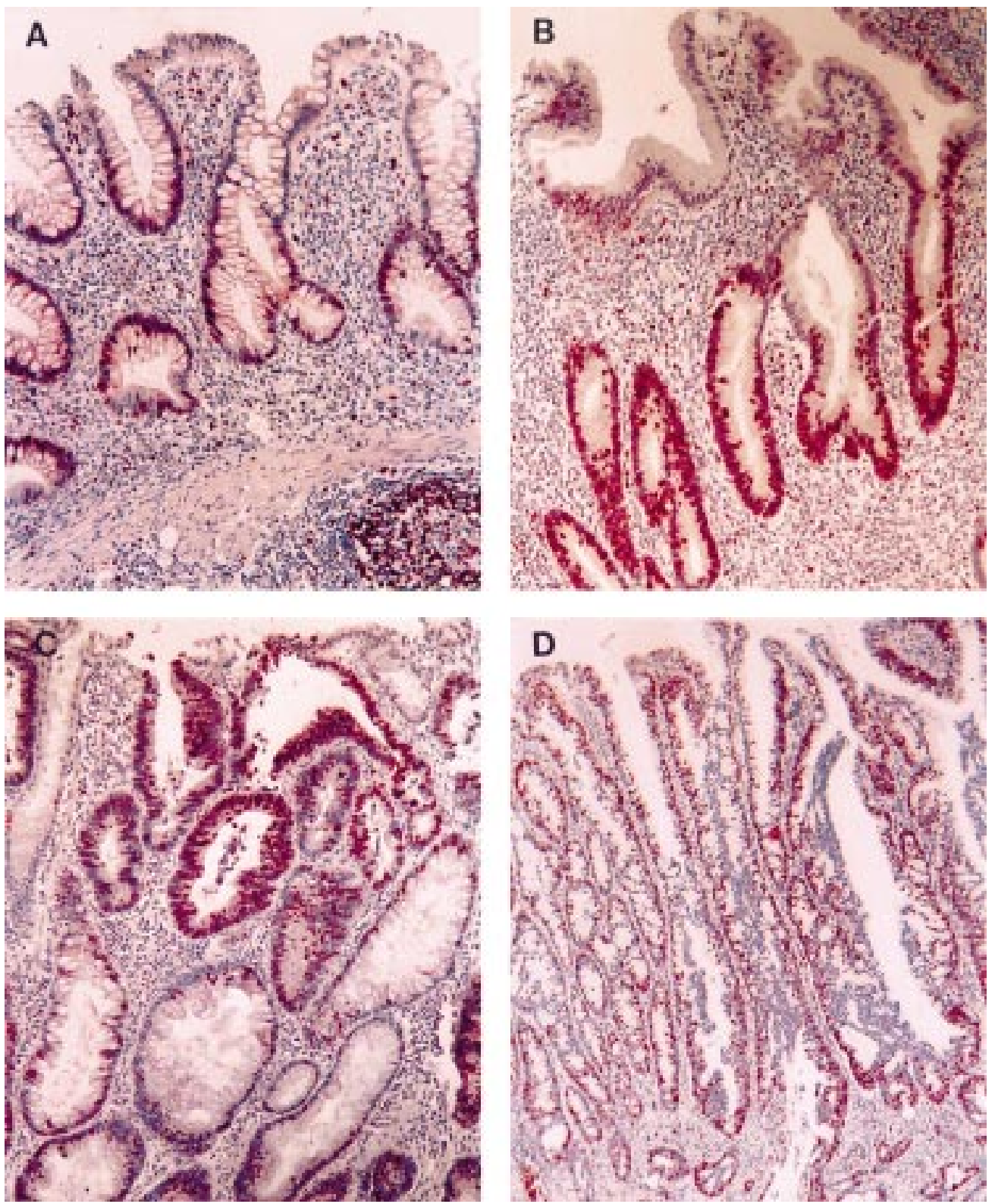

Figure 3 Different patterns of Ki-67 immunoreactivity. (A) Expanded basal zone in a section with regerative epithelium, with positive cells localised mainly to the bottom and middle section of the crypt. (B) Low grade dysplasia shows positive cells throughout the full length of the crypts, with expansion almost to the top, whereas in high grade dysplasia (C), positive cells are marked at the top of the mucosa and are scattered throughout the lesion. (D) In carcinoma, the cells are stained diffusely in all compartments of the crypts, as well as in the surface epithelium and the infiltrating crypts.

compared with normal epithelium and epithelium with regenerative changes ( $\mathrm{p}<0.001$ for each of the groups). Regenerative epithelium was not different from epithelium indefinite for dysplasia $(p \approx 1)$ with respect to $\mathrm{Ki}-67$ positivity.

In normal colonic mucosa, the predominant area of cell proliferation is localised to the lower one third of the crypts, cells then migrate from the base of the crypt upwards towards the luminal surface, where they are sloughed off. ${ }^{23}$ Deschner ${ }^{23}$ and Lipkin ${ }^{24}$ were the first to find abnormal, proliferative cellular lesions in colonic crypts in animal models and in humans with familial adenomatous polyposis, sporadic adenomas, and carcinomas, as well as in ulcerative colitis. ${ }^{25}$ They concluded that increased DNA synthesis might be an important step in a final common pathway leading to malignant transformation.

Biasco et al used tritiated thymidine in their cell kinetic studies of non-neoplastic rectal epithelium in patients with ulcerative colitis, and compared the results with those from patients with multiple sporadic adenomas and control subjects without colonic disease. ${ }^{26}$ The labelling index was similar in the three groups, but there was an overall increase in the pool of proliferating cells in patients with ulcerative colitis, especially in the upper $40 \%$ of the crypts. Alpers et al found that patients with long standing ulcerative colitis of more than 10 years duration showed a lack of inhibition by phosphodiesterase inhibitors on thymidine incorporation into DNA in cultured colonic tissue. ${ }^{27}$ They concluded that the duration of 
inflammation, rather than the extent of the disease within the colon, might be the more important factor leading to alteration in the control of DNA synthesis.

Several authors have found that the proliferative compartment in adenomas is expanded or misplaced, often reaching to the mucosal surface. ${ }^{1428}$ The proliferating cells are distributed uniformly throughout the section, with accentuation at the top.

Because there is an almost linear increase in the $\mathrm{Ki}-67$ reactivity from normal colorectal mucosa, through regeneration and various grades of dysplasia, to carcinoma (fig 2), our results clearly confirm the importance of increased cell proliferation for the progression to malignancy in this context. Furthermore, this process is also associated with a disruption of the normal structural organisation of the growth compartment, because most high grade dysplasias and all cancers had a diffuse staining pattern with scattered $\mathrm{Ki}-67$ positive cells (table 2; fig 3).

Several studies have shown that there is a very close association between Ki-67 immunoreactivity and the cell cycle. Expression appears in mid to late $\mathrm{G} 1$, rising through $S$ phase and G2 to reach a maximum during mitosis. ${ }^{29}$ After mitosis, the antigen is degraded rapidly, with a half life of an hour or less for the detectable antigen. ${ }^{30}$ There is strong evidence that $\mathrm{Ki}-67$ expression correlates with cell proliferation, although it consistently overestimates the growth fraction, as measured by $\mathrm{S}$ and G2 fractions. ${ }^{31}$

In the development of sporadic colorectal carcinomas, mutations in the tumour suppressor gene APC and in the protooncogene $\mathrm{Ki}$-ras are relatively early events that are assumed to be related to increased cell proliferation. ${ }^{32}$ However, because there are conflicting reports on the frequency of mutations within these genes during tumour development in ulcerative colitis, ${ }^{33-35}$ such mutations are not likely to be able to explain the increased proliferation in dysplastic development seen in ulcerative colitis. Putative candidate genes involved in this process would be those encoding cyclins, cyclin dependent kinases, and their inhibitors.

Two recent studies also used the MIB 1 antibody in formalin fixed, paraffin wax embedded colonic tissue to detect $\mathrm{Ki}-67$ positive nuclei in dysplastic and non-dysplastic lesions from patients with inflammatory bowel disease. ${ }^{18} 19$ Kullmann et al found labelling indices significantly higher in high grade dysplasia compared with low grade dysplasia in both upper and lower compartments, and significantly higher in the superficial part in sections "indefinite for dysplasia, probably positive", compared with normal colorectal tissue. ${ }^{18}$ This agrees with our results. However, they could not show significant differences between the group that were "indefinite for dysplasia, probably positive" and low grade dysplasia, assuming that this could be caused by active inflammation. In our sections from patients with long standing ulcerative colitis very few patients had active inflammation. In our study, cells were counted along whole crypts up to the mucosal surface, without dividing them into compartments. In long standing colitis, the mucosa is often atrophic, with branching and distorted crypts not reaching the muscularis mucosa. In our opinion, this makes division of the crypts difficult to perform in a reproducible way. The fact that we had similar Ki-67 indices in regenerative epithelium and epithelium indefinite for dysplasia, whereas the latter category had Ki-67 indices similar to low grade dysplasia when scored by Kullmann and colleagues, ${ }^{18}$ might be a result of consistent differences in the subclassification of dysplasias.

Noffsinger et al concluded that dysplastic lesions and carcinomas show a different $\mathrm{Ki}-67$ staining pattern to regenerative epithelium, with positive cells often confined to the top of the crypts, which might help to delineate areas of dysplasia where distinction from regeneration based on histopathology alone is difficult. ${ }^{19}$ We also investigated the staining pattern in our material and found similar distributions in high grade dysplasias and carcinomas, as did these authors, but almost all lesions in our material showed varying degrees of expanded basal zones, with the exception of the carcinomas. Therefore, we recommend that the total percentage of Ki-67 stained cells should also be taken into account.

Traditional histopathological evaluation of dysplasia in long standing ulcerative colitis is often difficult, especially when deciding whether a lesion shows low grade dysplasia, regenerative changes, or is indefinite for dysplasia. To assure the reproducibility of this method, we recommend that assessment of both the percentage of positive cells, and the staining pattern, should be made. Our study is too small to predict the outcome for any definite patient. A new study with a larger patient population might show whether $\mathrm{Ki}-67$ based classification of mucosal alterations related to long standing ulcerative colitis is a more objective way of predicting malignant transformation than traditional histopathological evaluation alone.

The authors thank The Norwegian Cancer Society for financial support, and Dr J Gerdes for providing the monoclonal antibody MIB 1 . We are also indebted to Ms A Cromarty and Ms G Gulbrandsen for technical help, and to Professor T Egeland for assistance with the statistical analysis.

1 Morson BD, Pang LSC. Rectal biopsy as an aid to cancer control in ulcerative colitis. Gut 1967;8:423-34.

2 Lennard-Jones JE, Morson BC, Ritchie JK, et al. Cancer in colitis: assessment of the individual risk by clinical and hiscolitis: assessment of the individual risk by clinical

3 Lennard-Jones JE, Melville DM, Morson BC, et al. Precancer and cancer in extensive ulcerative colitis: findings cer and cancer in extensive ulcerative colitis: findings
among 401 patients over 22 years. Gut 1990;31:800-6.

4 Gyde SN, Prior P, Allan RN, et al. Colorectal cancer in Gyde SN, Prior P, Allan RN, et al. Colorectal cancer in
ulcerative colitis: a cohort study of primary referrals from three centres. Gut 1988;29: 206-17.

5 Sugita A, Sachar DB, Bodian C, et al. Colorectal cancer in ulcerative colitis. Influence of anatomical extent and age at onset on colitis-cancer interval. Gut 1991;32:167-9.

6 Riddell RH, Goldman H, Ransohoff DF, et al. Dysplasia in inflammatory bowel disease: standardized classification with provisional clinical applications. Hum Pathol 1983;14: 931-68

7 Paganelli GM, Lalli E, Facchini A, et al. Flow cytometric and in vitro tritiated thymidine labeling in normal rectal mucosa of patients at high risk of colorectal cancer. $A m \mathcal{F}$ mucosa of patients at high risk

8 Matthew JA, Pell JD, Prior A, et al. Validation of a simple technique for the detection of abnormal mucosal cell replication in humans. Eur f Cancer Prev 1994;3:337-44. 
9 Gerdes J, Li L, Schlueter C, et al. Immunobiochemical and molecular biologic characterization of the cell proliferation-
associated nuclear antigen that is defined by monoclonal associated nuclear antigen that is defined by mor

10 Cattoretti G, Becker MHG, Key G, et al. Monoclonal antibodies against recombinant parts of the Ki-67 antigen (MIB 1 and MIB 3) detect proliferating cells in microwave-processed formalin-fixed paraffin sections. $\mathcal{F}$ Pathol 1992;168:357-63.

11 McCormick D, Chong H, Hobbs C, et al. Detection of the Ki-67 antigen in fixed and wax-embedded sections with the monoclonal antibody MIB 1. Histopathology 1993;22:35560.

12 Holt PR, Moss SF, Kapetanakis AM, et al. Is Ki-67 a better proliferative marker in the colon than proliferating cell nuclear antigen? Cancer Epidemiol Biomarkers Prev 1997;6: 131-5.

13 Shepherd NA, Richman PI, England J. Ki-67 derived proliferative activity in colorectal adenocarcinoma with prognostic correlations. F Pathol 1988;155:213-19.

14 Hoang C, Polivka M, Valleur P, et al. Immunohistochemical detection of proliferating cells in colorectal carcinomas and adenomas with the monoclonal antibody Ki-67. Prelimiadenomas with the monoclonal antibody
nary data. Virchows Arch 1989;414:423-8.

15 Johnston PG, O'Brien MJ, Dervan PA, et al. Immunohistochemical analysis of cell kinetic parameters in colonic adenocarcinomas, adenomas, and normal mucosa. Hum Pathol 1989;20:696-700.

16 Kubota Y, Petras RE, Easly KA, et al. Ki-67-determined growth fraction versus standard staging and grading parameters in colorectal carcinoma. Cancer 1992;70:2602-9.

17 Suzuki H, Matsumoto K, Terabe M. Ki-67 antibody labeling index in colorectal carcinoma. 7 Clin Gastroenterol 1992;15:317-20.

18 Kullmann F, Fadaie M, Gross V, et al. Expression of proliferating cell nuclear antigen (PCNA) and $\mathrm{Ki}-67$ in dysplasia in inflammatory bowel disease. Eur $\mathcal{f}$ Gastroenterol Hepatol 1996;8:371-9.

19 Noffsinger AE, Miller MA, Cusi MV, et al. The pattern of cell proliferation in neoplastic and nonneoplastic lesions of cell proliferation in neoplastic and nonneop

20 Stave R, Brandtzaeg P. Fluorescence staining of gastric mucosa. A study with special reference to parietal cells. Scand $\mathcal{F}$ Gastroenterol 1977;12:885-91.

21 SAS Institute Inc. SAS/STAT software: changes and enhancements through release 6.11. Cary, NC: SAS Institute Inc, 1996.
22 Landis JR, Koch GG. The measurement of observer agreement for categorical data. Biometrics 1977;33:159-74.

23 Deschner EE. Early proliferative changes in gastrointestinal neoplasia. Am f Gastroenterol 1982;77:207-11.

24 Lipkin $M$. Phase 1 and phase 2 proliferative lesions of colonic epithelial cells in diseases leading to colonic cancer. Cancer 1974;34:878-88.

25 Deschner EE, Winawer SJ, Katz S, et al. Proliferative defects in ulcerative colitis patients. Cancer Invest 1983;1:41-7.

26 Biasco G, Paganelli GM, Miglioli M, et al. Rectal cell proliferation and colon cancer risk in ulcerative colitis. Cancer Res 1990;50:1156-9.

27 Alpers DH, Philpott G, Grimme NL, et al. Control of thymidine incorporation in mucosal explants from patients with chronic ulcerative colitis. Gastroenterology 1980;78: 470-8.

28 Risio M, Lipkin M, Candelaresi GL, et al. Correlations between rectal mucosa cell proliferation and the clinical and pathological features of nonfamilial neoplasia of the large intestine. Cancer Res 1991;51:1917-21.

29 Gerdes J, Lemke H, Baisch H, et al. Cell cycle analysis of a cell proliferation-associated human nuclear antigen defined by the monoclonal antibody Ki-67. F Immunol 1984;133: 1710-15.

30 Bruno S, Darzynkiewicz Z. Cell cycle dependent expression and stability of the nuclear protein detected by Ki-67 antibody in HL-60 cells. Cell Prolif 1992;25:31-40.

31 Scott RJ, Hall PA, Haldane JS, et al. A comparison of immunohistochemical markers of cell proliferation with experimentally determined growth fraction. F Pathol 1991; 165:173-8.

32 Fearon ER, Vogelstein B. A genetic model for colorectal tumorigenesis. Cell 1990;61:759-67.

33 Burmer GC, Levine DS, Kulander BG, et al. C-ki-ras mutations in chronic ulcerative colitis and sporadic colon carcinoma. Gastroenterology 1990;99:416-20.

34 Greenwald BD, Harpaz N, Yin J, et al. Loss of heterozygosity affecting the $\mathrm{p} 53, \mathrm{Rb}$, and mcc/apc tumor suppressor gene loci in dysplastic and cancerous ulcerative colitis. Cancer Res 1992;52:741-5.

35 Kern SE, Redston M, Seymour AB, et al. Molecular genetic profiles of colitis-associated neoplasms. Gastroenterology 1994;107:420-8. 